\title{
Images: Monolobar Caroli's disease
}

\author{
Sachit K Verma, Donald G Mitchell \\ Department of Radiology, Thomas J efferson University Hospital, Philadelphia 19107, USA
}

Correspondence: Dr. Sachit K. Verma, Department of Radiology, Thomas Jefferson University Hospital Philadelphia, PA 19107 USA.

E-mail: medskv@yahoo.com

Key words: Caroli's disease; CT; MRCP

Caroli's disease is the eponymous designation for congenital nonobstructive dilatation of the large intrahepatic bile ducts. ${ }^{[1]}$ This rare and incompletely delineated entity was first described by Caroli et al. in 1958. ${ }^{[2]}$ Caroli's disease may be multifocal and diffuse or may be localized to a lobe or segment of the liver, usually the left. Most cases are associated with congenital hepatic fibrosis. Medullary sponge kidneys may occur occasionally. ${ }^{[3]}$ The incidence of focal monolobar Caroli's disease is rare; less than $20 \%$ of cases are reported in literature ${ }^{[4]}$ The use of noninvasive techniques such as USG, CT scan, isotope scan, and magnetic resonance cholangiopancreatography (MRCP) helps in the diagnosis. Invasive techniques such as endoscopic retrograde cholangiopancreatography (ERCP) and percutaneous transhepatic cholangiography (PTC) are, therefore, now rarely required. Histopathological examination of the resected specimen is still the gold standard for diagnosing monolobar Caroli's disease.

\section{Case Report}

A 23-year-old man presented with right upper quadrant abdominal pain of three months' duration. The pain was intermittent and cramping and occurred after meals. He denied having had any fever, chills, or malaise. On physical examination, there was no fever or evidence of jaundice. The liver was normal. The spleen was not palpable. Liver function tests such as bilirubin, prothrombin time, alkaline phosphatase, and albumin were normal; levels of serum glutamate oxaloacetate transaminase (SGOT) and serum glutamate pyruvate transaminase (SGPT) were mildly elevated. The renal parameters were normal.

Color Doppler USG showed saccular dilatation of the intrahepatic ducts, with abnormal flow signal within [Figure 1A]. Contrast-enhanced CT scan of the abdomen showed an abnormal area consisting of multiple tortuous, tubular structures in the posterior segment of the right lobe of the liver, some of which contained portal radicles (central dot

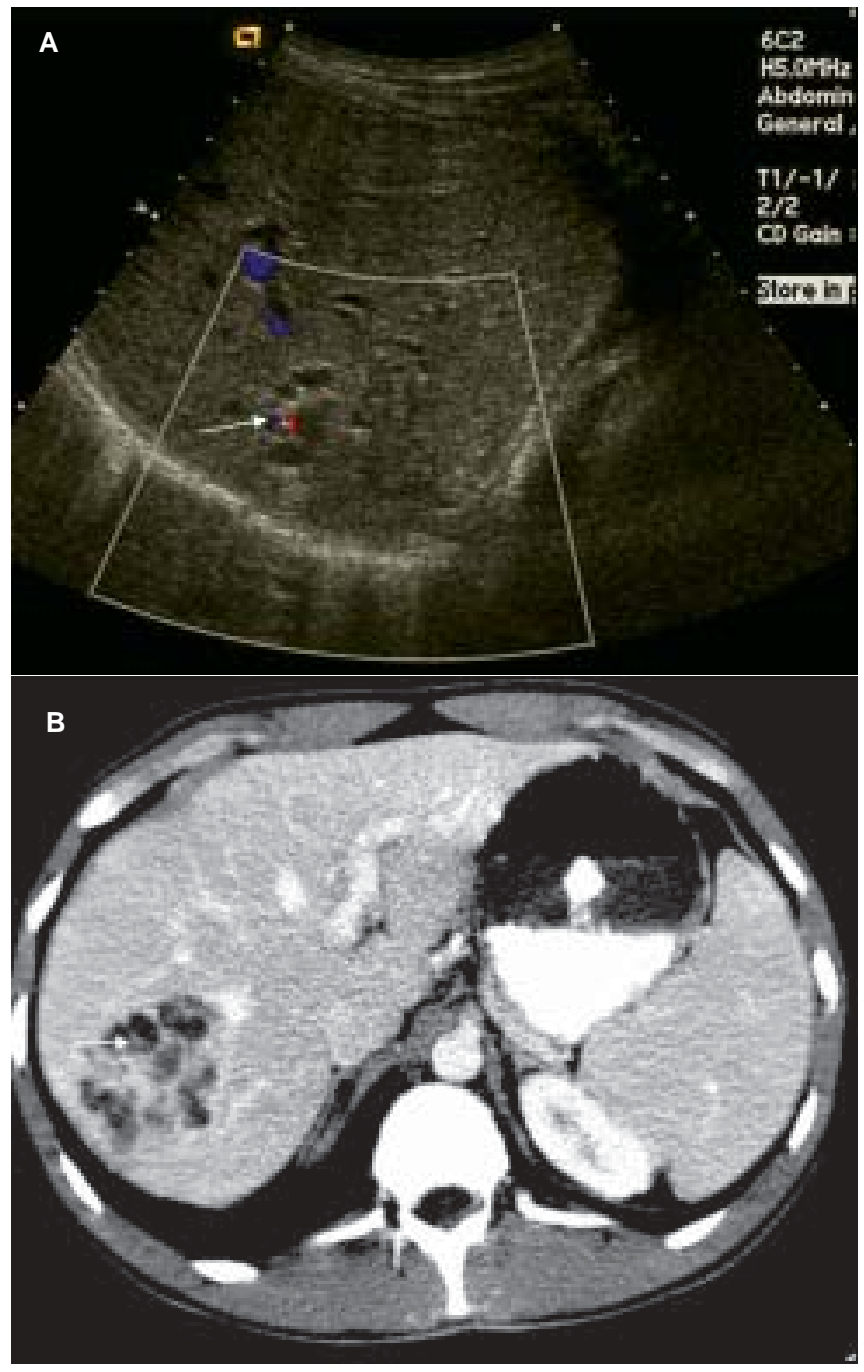

Figure 1 (A, B): Axial color Doppler USG (A) image of the liver reveals multiple, small, rounded anechoic structures, corresponding to the saccular dilatations of the biliary tree with abnormal flow signal within (arrow). Contrast-enhanced CT scan (B) shows a cluster of serpiginous, abnormal hypodense structures. Within the hypodense regions, there are punctate foci of high density (central dot sign), representing portal radicles (arrow) 
sign) [Figure 1B]. MRI of the abdomen showed a rounded tangle of dilated nonenhancing cystic tubular structures [Figure 2A] in the right lobe of the liver, with focal cystic dilatation of the intrahepatic bile ducts and a normal common bile duct [Figure 2]. In all the studies, there was no evidence of portal hypertension. The common bile duct and gall bladder were normal.

These findings suggested a segmental anomaly of dilated ducts. The patient underwent a right hepatic lobectomy subsequently. Sectioning through the parenchyma revealed a $5.1 \times 5.0 \times 4.9 \mathrm{~cm}$ irregular area of cystic, dilated ducts, which were filled with thick, green material. Histological examination of the cysts showed hyperplasia of the biliary epithelium but no dysplasia [Figure 3]. The typical stromal component of a cystadenoma was not present. There was no evidence of malignancy. This appearance was consistent with right-sided monolobar Caroli's disease.

\section{Discussion}

Caroli's disease results from a bile duct malformation, which involves neonatal occlusion of the hepatic artery, leading to bile duct ischemia, cystic dilatation, and abnormal growth rate of the developing biliary epithelium and its supporting connective tissue. ${ }^{[5]}$ Incomplete resorption of the circular plates leads to the formation of multiple primitive bile ducts surrounding portal vein radicles. ${ }^{[1]}$ It is believed to have an autosomal recessive inheritance pattern and usually presents with intermittent abdominal pain and hepatomegaly. ${ }^{[6]}$ Cholangitis, cholelithiasis, biliary abscess, septicemia, liver cirrhosis, and cholangiocarcinoma (upto $7 \%)$ are all potential complications. ${ }^{[6]}$

The dilated sacculi or cystic spaces are anechoic on USG and hypodense on CT scan. The fibrovascular bundles, containing portal vein radicles and a branch of the hepatic artery bridging the saccule, appear as a central dot or a linear structure on $\mathrm{CT}$, enhancing with contrast. ${ }^{[7]}$ This 'central dot sign' is also seen on USG. ${ }^{[8]} \mathrm{CT}$ scan demonstrates the extent of disease well. MRI provides information about the severity, location, and extent of liver involvement. In our case, this information was useful for planning the correct therapeutic strategy.

The appearance of monolobar Caroli's disease may be confused with primary sclerosing cholangitis, recurrent pyogenic cholangitis, polycystic liver disease, choledochal cyst, biliary papillomatosis and, occasionally, focal obstructive biliary dilatation. ${ }^{[9]}$ MRI is superior to USG and $\mathrm{CT}$ scan in its ability to differentiate between these conditions. ${ }^{[10]}$

Direct cholangiography is rarely necessary for an accurate diagnosis ${ }^{[11]}$ and both ERCP and PTC carry a high risk of bacterial cholangitis. ${ }^{[12]}$ Histological examination of the
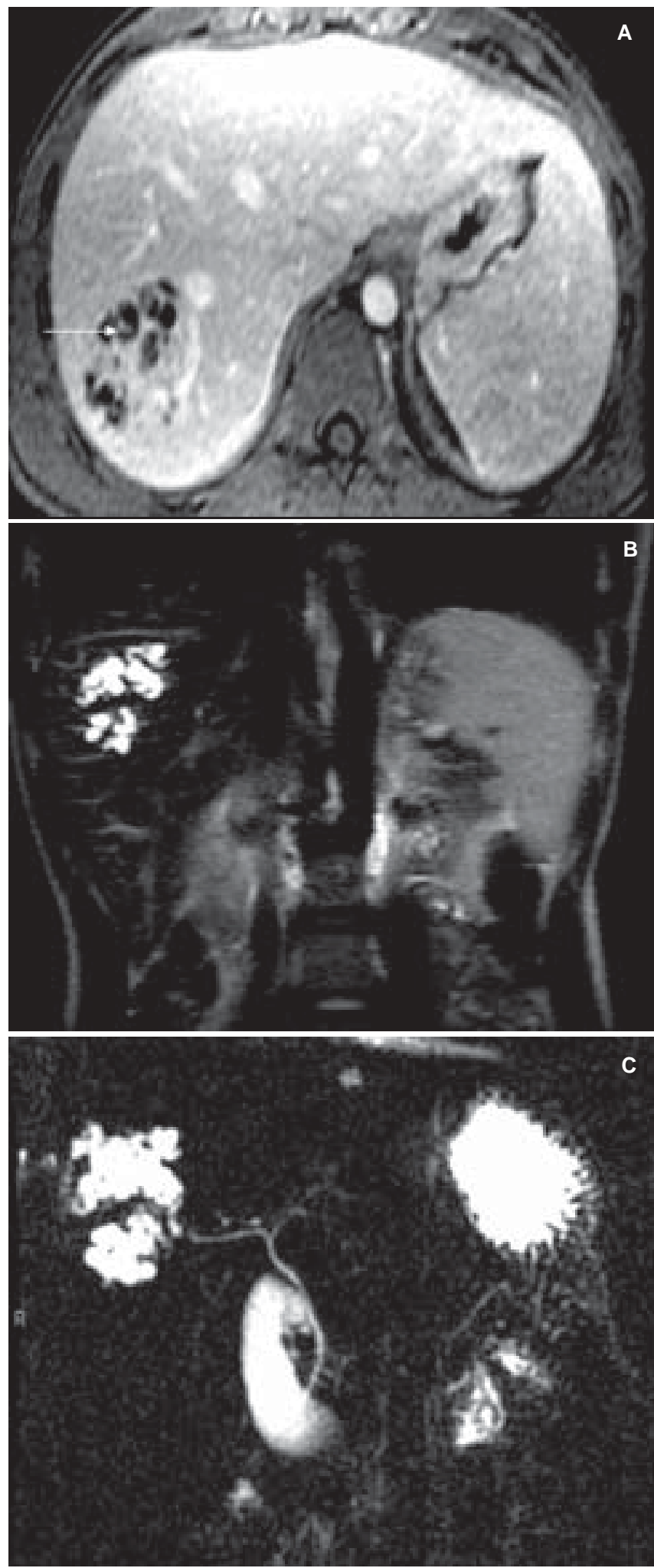

Figure 2 (A-C): Axial contrast-enhanced fast gradient-echo 3D image (A) in the blood-pool phase shows multiple hypointense cystic lesions in the right lobe of the liver with fibrovascular bundles within (arrow). Coronal single-shot fast spin-echo (TE $=180 \mathrm{msec}$ ) image $(B)$ demonstrates focal hyperintense cystic dilatation of the biliary ducts. Coronal single slice (thickness $=40 \mathrm{~mm}$ ) MRCP image $(C)$ reveals focal cystic ectasia of the intrahepatic bile ducts, with a normal common bile duct and gall bladder 


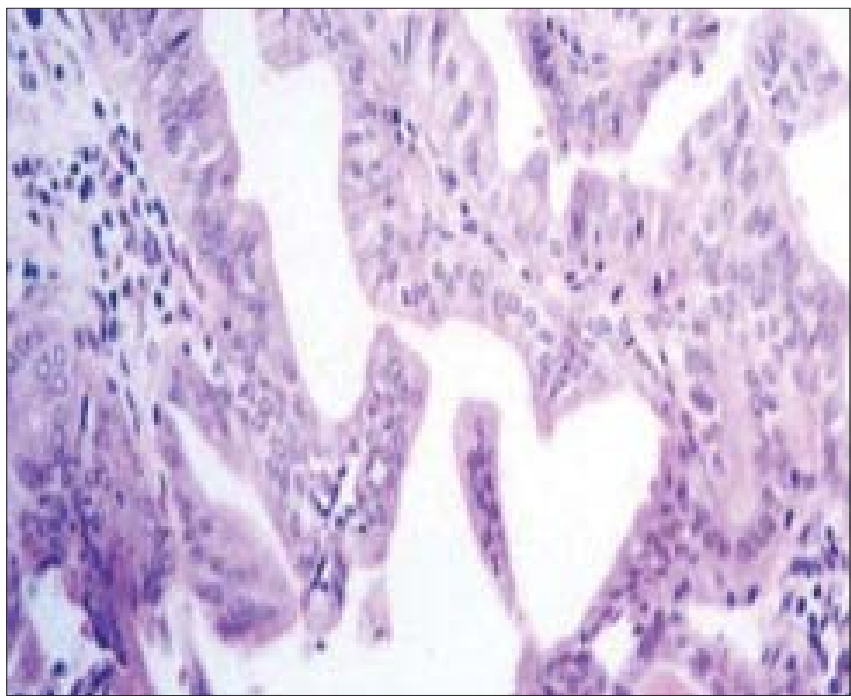

Figure 3: Histopathology shows hyperplastic epithelium of the intrahepatic biliary ducts (H\&E, x40)

resected specimen may be valuable and will confirm the diagnosis in equivocal cases. Benign lesions do not show dysplasia of the epithelium of the biliary ducts; instead, hyperplasia and ulceration are the common pathological manifestations. ${ }^{[13]}$

The aim of therapy is to decrease the morbidity and mortality associated with the recurrent cholangitis, hepatic abscess, and cholangiocarcinoma. Lobar or segmental resection may lead to a complete cure when the disease is confined to one lobe. In our patient, lobectomy relieved symptoms. In the diffuse form, conservative management and/or biliary drainage procedures are effective in decompressing the biliary tract and avoiding complications. Orthotopic liver transplantation is the best alternative, with excellent longterm results. ${ }^{[13]}$

\section{References}

1. Desmet VJ. Congenital diseases of intrahepatic bile ducts: Variations on the theme ductal plate malformation. Hepatology 1992;16:1069-83.

2. Caroli J, Soupault R, Kossakowski J, Plocker L, Paradowska M. The polycystic conge' nitale dilatation of bile ducts intrahe' patiques: Test classification. Semin Hop Paris 1958;34:488-95.

3. Mall JC, Ghahremani GG, Boyer JL. Caroli's disease associated with congenital hepatic fibrosis and renal tubular ectasia. Gastroenterology 1974;66:1029-35.

4. Giovanardi RO. Monolobar Caroli's disease in an adult: Case report. Hepatogastroenterology 2003;50:2185-7.

5. Marchal GJ, Desmet VJ, Proesmans WC, Moerman PL, Van Roost WW, Van Holsbeeck MT, et al. Caroli disease: High-frequency US and pathologic findings. Radiology 1986;158:507-11.

6. Taylor AC, Palmer KR. Caroli's disease. Eur J Gastroenterol Hepatol 1998;10:105-8.

7. Choi BI, Yeon KM, Kim SH, Han MC. Caroli disease: Central dot sign in CT. Radiology 1990;174:161-3.

8. Seth AK, Chawla Y, Dhiman RK, Gupta S, Suri S, Dilawari JB. Caroli's disease: A central dot means a lot. Trop Gastroenterol 1997;18:165-6.

9. Mortelé KJ, Ros PR. Cystic focal liver lesions in the adult: Differential CT and MR imaging features. Radiographics 2001;21:895-910.

10. Pavone P, Laghi A, Catalano C, Materia A, Basso N, Passariello R. Caroli's disease: Evaluation with MR cholangiopancreatography (MRCP). Abdom Imaging 1996;21:117-9.

11. Lucaya J, Gomez JL, Molino C, Atienza JG. Congenital dilatation of the intrahepatic bile ducts (Caroli's disease). Radiology 1978;127:746.

12. Missavage AE, Sugawa C. Caroli's disease: Role of endoscopic retrograde cholangiopancreatography. Am J Gastroenterol 1983;78:815-7.

13. Dagli U, Atalay F, Sasmaz N, Bostanoðlu S, Temuçin G, Sahin B. Caroli's disease: 1977- 1995 experiences. Eur J Gastroenterol Hepatol 1998;10:109-12.

Source of Support: Nil, Conflict of Interest: None declared. 